\title{
Psychometrics of health-related quality of life questionnaires in bronchiectasis: a systematic review and meta-analysis
}

\author{
Rebecca H. McLeese $\mathbb{B}^{1}$, Arietta Spinou $\mathbb{B}^{2}$, Zina Alfahl ${ }^{1,3}$, Michail Tsagris ${ }^{4}$, J. Stuart Elborn ${ }^{5}$, \\ James D. Chalmers ${ }^{6}$, Anthony De Soyza $0^{7}$, Michael R. Loebinger ${ }^{8}$, Surinder S. Birring ${ }^{9}$, \\ Konstantinos C. Fragkos $^{10}$, Robert Wilson ${ }^{8}$, Katherine $0^{\prime}$ Neill $^{1,5,11}$ and Judy M. Bradley ${ }^{1,5,11}$
}

\begin{abstract}
${ }^{1}$ The Wellcome Trust-Wolfson Northern Ireland Clinical Research Facility, School of Medicine, Dentistry and Biomedical Sciences, Queen's University Belfast, Belfast, UK. ${ }^{2}$ Population Health Sciences, Faculty of Life Sciences and Medicine, King's College London, London, UK. ${ }^{3}$ School of Pharmacy, Queen's University Belfast, Belfast, UK. ${ }^{4}$ Dept of Economics, University of Crete, Rethymnon, Greece. ${ }^{5}$ Wellcome-Wolfson Institute for Experimental Medicine, School of Medicine, Dentistry and Biomedical Sciences, Queen's University Belfast, Belfast, UK. ${ }^{6}$ Scottish Centre for Respiratory Research, University of Dundee, Dundee, UK. ${ }^{7}$ Respiratory Dept, Institute of Cellular Medicine, Newcastle University and Freeman Hospital, Sir William Leech Research Centre, Newcastle upon Tyne, UK. ${ }^{8}$ Host Defence Unit, Royal Brompton Hospital, London, UK. ${ }^{9}$ Centre for Human and Applied Physiological Sciences, School of Basic and Medical Biosciences, Faculty of Life Sciences and Medicine, King's College London, London, UK. ${ }^{10}$ Division of Medicine, University College London, London, UK. ${ }^{11}$ These two authors are co-senior authors.
\end{abstract}

Corresponding author: Judy M. Bradley (Judy.Bradley@qub.ac.uk)

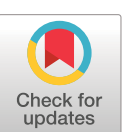

Copyright @The authors 2021

This version is distributed under the terms of the Creative Commons Attribution Licence 4.0.

This article has supplementary material available from erj.ersjournals.com

Received: 6 Jan 2021 Accepted: 2 April 2021

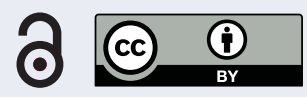

Shareable abstract (@ERSpublications)

The psychometric properties of health-related quality of life questionnaires should inform the selection of patient-reported outcomes in bronchiectasis clinical trials https://bit.ly/3wQuSrm

Cite this article as: McLeese RH, Spinou A, Alfahl Z, et al. Psychometrics of health-related quality of life questionnaires in bronchiectasis: a systematic review and meta-analysis. Eur Respir J 2021; 58: 2100025 [DOI: 10.1183/13993003.00025-2021].

Abstract

Introduction Understanding the psychometric properties of health-related quality of life (HRQoL) questionnaires can help inform selection in clinical trials. Our objective was to assess the psychometric properties of HRQoL questionnaires in bronchiectasis using a systematic review and meta-analysis of the literature.

Methods A literature search was conducted. HRQoL questionnaires were assessed for psychometric properties (reliability, validity, minimal clinically important difference (MCID) and floor/ceiling effects). Meta-analyses assessed the associations of HRQoL with clinical measures and responsiveness of HRQoL in clinical trials.

Results 166 studies and 12 HRQoL questionnaires were included. The Bronchiectasis Health Questionnaire (BHQ), Leicester Cough Questionnaire (LCQ), Chronic Obstructive Pulmonary Disease (COPD) Assessment Test (CAT) and Medical Outcomes Study 36-item Short-Form Health Survey (SF-36) had good internal consistency in all domains reported (Cronbach's $\alpha \geqslant 0.7$ ) across all studies, and the Quality of Life-Bronchiectasis (QOL-B), St George's Respiratory Questionnaire (SGRQ), Chronic Respiratory Disease Questionnaire (CRDQ) and Seattle Obstructive Lung Disease Questionnaire (SOLQ) had good internal consistency in all domains in the majority of (but not all) studies. BHQ, SGRQ, LCQ and CAT had good test-retest reliability in all domains reported (intraclass correlation coefficient $\geqslant 0.7$ ) across all studies, and QOL-B, CRDQ and SOLQ had good test-retest reliability in all domains in the majority of (but not all) studies. HRQoL questionnaires were able to discriminate between demographics, important markers of clinical status, disease severity, exacerbations and bacteriology. For HRQoL responsiveness, there was a difference between the treatment and placebo effect.

Conclusions SGRQ was the most widely used HRQoL questionnaire in bronchiectasis studies and it had good psychometric properties; however, good psychometric data are emerging on the bronchiectasisspecific HRQoL questionnaires QOL-B and BHQ. Future studies should focus on the medium- to longterm test-retest reliability, responsiveness and MCID in these HRQoL questionnaires which show potential in bronchiectasis. 


\section{Introduction}

Bronchiectasis is associated with significant morbidity and substantial healthcare utilisation. There are no licensed medications available and current management guidelines have emphasised the importance of randomised controlled trials to direct care [1, 2]. Evidence from recent clinical trials supports the use of some medications, including macrolides, inhaled antibiotics and novel anti-inflammatory therapies [3-5]. However, a number of other clinical trials have failed to demonstrate a clinically important or statistically significant change in their primary end-points, including measures of quality of life (QoL) [6-9]. The reasons for this are multifactorial, including ineffective treatments, lack of consideration of treatable traits, and underlying physiology and microbiome, but also the inability of interventions to affect specific domain scores of health-related QoL (HRQoL) questionnaires and a lack of bronchiectasis-specific, clinically validated outcome measures. The need for robust outcome measures in clinical trials has been emphasised by regulators $[10,11]$, with particular emphasis on tools used to measure patient-reported outcomes (PROs).

In 2016, SpINou et al. [12] published a systematic review summarising data from 57 studies across nine HRQoL questionnaires in bronchiectasis. They concluded that most of the HRQoL questionnaires had good reliability and validity. The responsiveness of HRQoL questionnaires ranged from trivial to large and there were some differences between questionnaires in their association with clinical measures such as demographics, symptoms, disease severity, lung function, bacteriology and healthcare utilisation.

Since the SpINou et al. [12] systematic review was completed, and based on a recent search of the literature, 1574 new bronchiectasis publications have been identified with data from a broad range of HRQoL questionnaires. For example, a recent large clinical trial, RESPIRE-1 [13], reported a significant change in the St George's Respiratory Questionnaire (SGRQ) but not in Quality of Life-Bronchiectasis (QOL-B) from baseline to end of treatment; discordance between multiple questionnaires causes significant challenges with regulatory approval. The aim of the current systematic review was to determine the psychometric properties of all HRQoL questionnaires available for use in bronchiectasis and to perform a meta-analysis of responsiveness in randomised controlled trials. These data will assist investigators and clinicians in their selection of HRQoL PROs and inform the design of clinical trials in bronchiectasis.

Methods

Protocol and registration

The methods for this systematic review and meta-analysis are described in a protocol registered with the International Prospective Register of Systematic Reviews (PROSPERO: CRD42019146181). Findings were reported according to the PRISMA (Preferred Reporting Items for Systematic Reviews and Meta-Analyses) guidelines [14] and the Scottish Intercollegiate Guidelines Network methodology checklist for systematic reviews and meta-analyses [15].

\section{Study eligibility criteria}

Empirical studies of adult patients ( $\geqslant 18$ years old) with bronchiectasis, studies reporting on the psychometric properties of HRQoL, and studies reporting on the association between HRQoL and clinical measures were included. In all studies, the diagnosis of bronchiectasis was established by clinical and/or radiological features. Only studies published in the English language were included. Reviews and protocols were excluded. Inclusion and exclusion criteria are listed in full in supplementary table E1.

The following psychometric properties of HRQoL questionnaires were assessed: reliability (internal consistency and test-retest reliability (including timescale of test-retest)), validity (translational validity and discriminant validity), correlations of HRQoL with clinical measures, responsiveness including effect size and minimal clinically important difference (MCID), floor/ceiling effects, and missing data [16, 17]. The psychometric properties assessed are listed in full in supplementary table E1.

\section{Data sources and searches}

Two authors (R.H.M. and Z.A.) independently searched the following databases: Embase, PubMed, MEDLINE, PsycINFO and the Cochrane Library (for search strategy, see supplementary table E2). Further searches were performed including the names of the identified HRQoL questionnaires. Reviews and references from included studies were manually searched to identify additional studies. The literature search included all studies published between 6 November 2014 and 31 December 2020. SpINou et al. [12] reported on all relevant studies published prior to 6 November 2014. All relevant studies from the current literature search and the prior review by SpINou et al. [12] were included in our review. 
Study selection, data extraction and quality assessment

R.H.M. and Z.A. independently screened articles by title and abstracts against the inclusion criteria, and full text was subsequently reviewed. Abstracts were considered for inclusion if there was adequate information regarding study methods and results. R.H.M. and Z.A. extracted the data, including: author, year of publication, study aim, sample size, disease aetiology, age, gender, lung function (forced expiratory volume in $1 \mathrm{~s}\left(\mathrm{FEV}_{1}\right) \%$ predicted), HRQoL questionnaire used and its psychometric properties. The quality of studies was assessed by R.H.M. and Z.A. using a modified tool by Swigris et al. [18] (appendix 1 in the supplementary material). Multiple articles from the same study were considered as a single study. The original article was cited in most cases, except when a subsequent publication presented new data and/ or performed secondary analyses on the original study data; in this case the data was attributed to the later publication. J.B., K.O.N. and A.S. were consulted to resolve any disagreements. For author roles, see appendix 2 in the supplementary material.

\section{Statistical analysis}

Quantitative analysis was performed with R version 3.6.3 (R Foundation for Statistical Computing, Vienna, Austria). Internal consistency was reported as Cronbach's $\alpha$ coefficient, with values $\geqslant 0.7$ considered acceptable [19]. Test-retest reliability was reported with intraclass correlation coefficient (ICC), with values $\geqslant 0.7$ considered acceptable for reproducibility [19, 20].

Meta-analysis was performed to assess associations between HRQoL questionnaires and clinical measures. Correlation coefficients (r) were extracted from the collected studies whenever this information was supplied. In cases where only p-values or test statistics (t-values, Cohen's d, F-values or Chi-squared values) were available, correlation coefficients were extracted according to formulas suggested by Rosenthal et al. [21]. The strength of association was assessed using the same categorisation as in SPINOU et al. [12]: $|\mathrm{r}|<0.4$ (weak), $0.4<|\mathrm{r}|<0.7$ (moderate) and $|\mathrm{r}|>0.7$ (strong). The statistical significance of $\mathrm{r}$ was evaluated at the $5 \%$ level of significance $(\alpha=0.05)$.

Meta-analysis was performed to determine the effect of bronchiectasis treatments versus placebo on HRQoL. The treatment, placebo and total combined effect sizes were classified according to the questionnaire. In addition, effect sizes were presented cumulatively by year, highlighting the direction of the effect size and whether there was stability through the time course.

Heterogeneity between studies was tested with the standard Chi-squared test. A random effects model was used to determine effect sizes with $95 \%$ confidence intervals. We tested for asymmetry of funnel plots to determine the presence of publication bias. Questionnaires with $<15 \%$ floor/ceiling effects were defined as meeting standards and $>15 \%$ floor/ceiling effects were defined as failed to have met standards.

Supplementary methodology is presented in appendix 3 in the supplementary material.

Results

Study characteristics

Study selection

The PRISMA flowchart shows the selection process of new studies, including reasons for study exclusion (figure 1).

\section{Study overview and study quality}

161 out of 166 studies included in the review were prospective. 71 studies reported cross-sectional findings and 37 studies reported longitudinal findings. 53 studies reported on clinical trials. Studies met a mean (range) of $45 \%$ (5-81\%) of the quality criteria. The quality of the new studies published was similar to those presented in the prior review by SpINou et al. [12] (figure 2). The studies published in abstract form met fewer of the criteria for quality domains: mean (range) quality score 30\% (5-57\%). No study met the criteria for all quality domains. The full list of studies is included in the References list and supplementary material.

\section{Patient characteristics}

The studies included 47540 patients with bronchiectasis. Mean (range) age was 60 (38-74) years and 62\% (24-91\%) were female. Eight studies recruited participants during an exacerbation [22-29], while all other studies recruited clinically stable patients. 115 studies required computed tomography scan findings to confirm the bronchiectasis diagnosis, while all other studies required a clinician diagnosis and symptoms consistent with bronchiectasis. Clinical characteristics of patients included in the newly identified studies are summarised in supplementary table E3. 


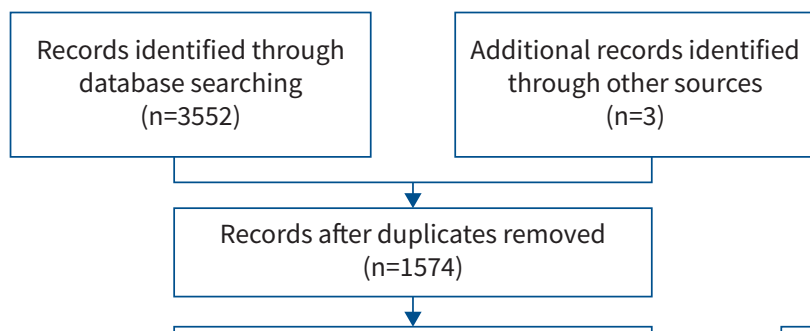

\begin{tabular}{|c|c|}
\hline $\begin{array}{l}\text { Abstracts reviewed } \\
\qquad(n=1574)\end{array}$ & $\begin{array}{l}\text { Records excluded: did not meet the } \\
\text { inclusion criteria }(n=1262)\end{array}$ \\
\hline \multicolumn{2}{|l|}{$\downarrow$} \\
\hline $\begin{array}{l}\text { Full texts assessed for eligibility } \\
\qquad(n=312)\end{array}$ & \multirow{3}{*}{$\begin{array}{l}\text { Records excluded, with reasons }(n=183) \text { : } \\
\text { Not English }(n=4) \\
\text { Not bronchiectasis population ( } n=34) \\
\text { No HRQoL data }(n=75) \\
\text { Abstracts already covered by full } \\
\text { manuscripts ( } n=54) \\
\text { Not primary research, e.g. review } \\
\text { papers, protocols }(n=16)\end{array}$} \\
\hline$\downarrow$ & \\
\hline $\begin{array}{l}\text { Studies included: } \\
\text { Observational studies: } n=55 \text { full texts, } \\
35 \text { abstracts }(n=76)^{\#} \\
\text { Clinical trials: } n=23 \text { full texts, } \\
16 \text { abstracts }(n=33)^{\#}\end{array}$ & \\
\hline \multicolumn{2}{|l|}{$\downarrow$} \\
\hline $\begin{array}{l}\text { New studies included in } \\
\text { the systematic review } \\
\qquad(n=109)\end{array}$ & \\
\hline
\end{tabular}

b)

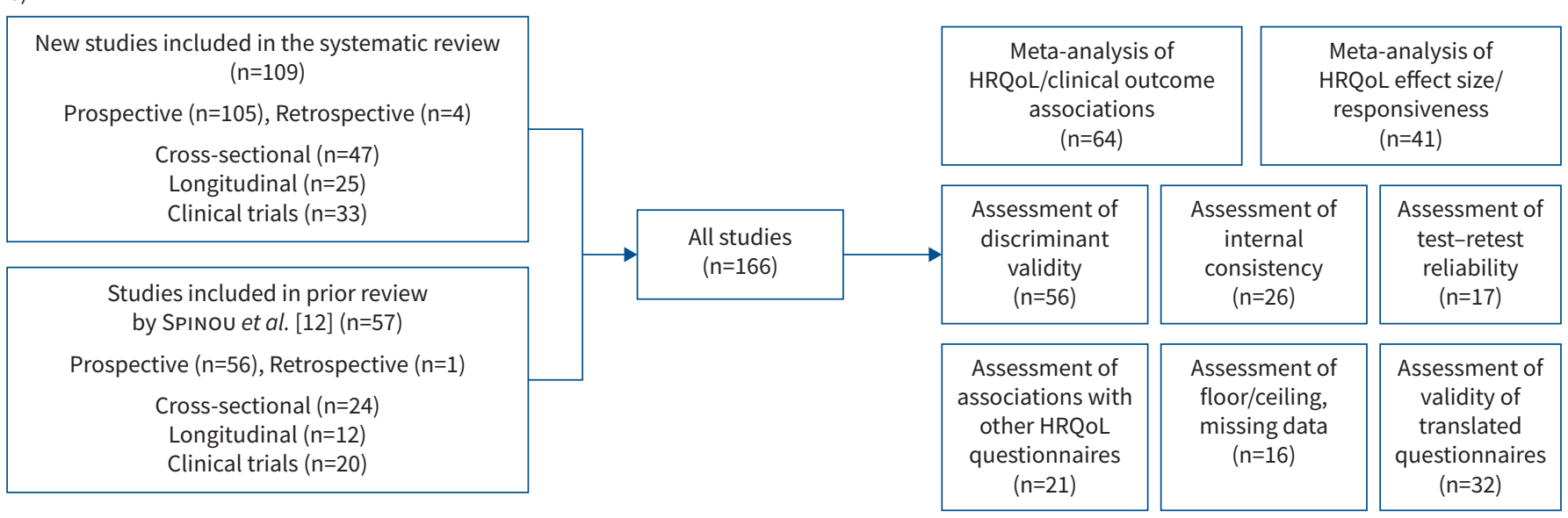

FIGURE 1 a) Flowchart of new studies (published between 6 November 2014 and 31 December 2020) included in the review based on the PRISMA (Preferred Reporting Items for Systematic Reviews and Meta-Analyses) protocols. HRQoL: health-related quality of life. \#: studies with multiple articles were subsequently combined and considered as a single study. The search yielded 1574 new studies. After screening the titles, abstracts and full texts when necessary, 109 new studies fulfilled the eligibility criteria for inclusion in the systematic review. b) Flowchart of total studies included in the review (studies reported by Sinou et al. [12] and studies published between 6 November 2014 and 31 December 2020).

HRQoL questionnaires

Overview

The 12 HRQoL questionnaires used were categorised as bronchiectasis-specific, respiratory-specific or generic. Bronchiectasis-specific questionnaires were QOL-B and Bronchiectasis Health Questionnaire (BHQ). The respiratory-specific questionnaires were SGRQ, Leicester Cough Questionnaire (LCQ), Chronic Obstructive Pulmonary Disease (COPD) Assessment Test (CAT), Chronic Respiratory Disease Questionnaire (CRDQ), Sino-Nasal Outcome Test-20 (SNOT-20), Cough Quality of Life Questionnaire (CQLQ) and Seattle Obstructive Lung Disease Questionnaire (SOLQ). The generic questionnaires were the Medical Outcomes Study 36-item Short-Form Health Survey (SF-36), EuroQoL five-dimension five-level (EQ-5D-5L) and Nottingham Health Profile (NHP) (full descriptions in appendix 4 in the supplementary material). The number of studies that used the different HRQoL questionnaires is shown in supplementary 


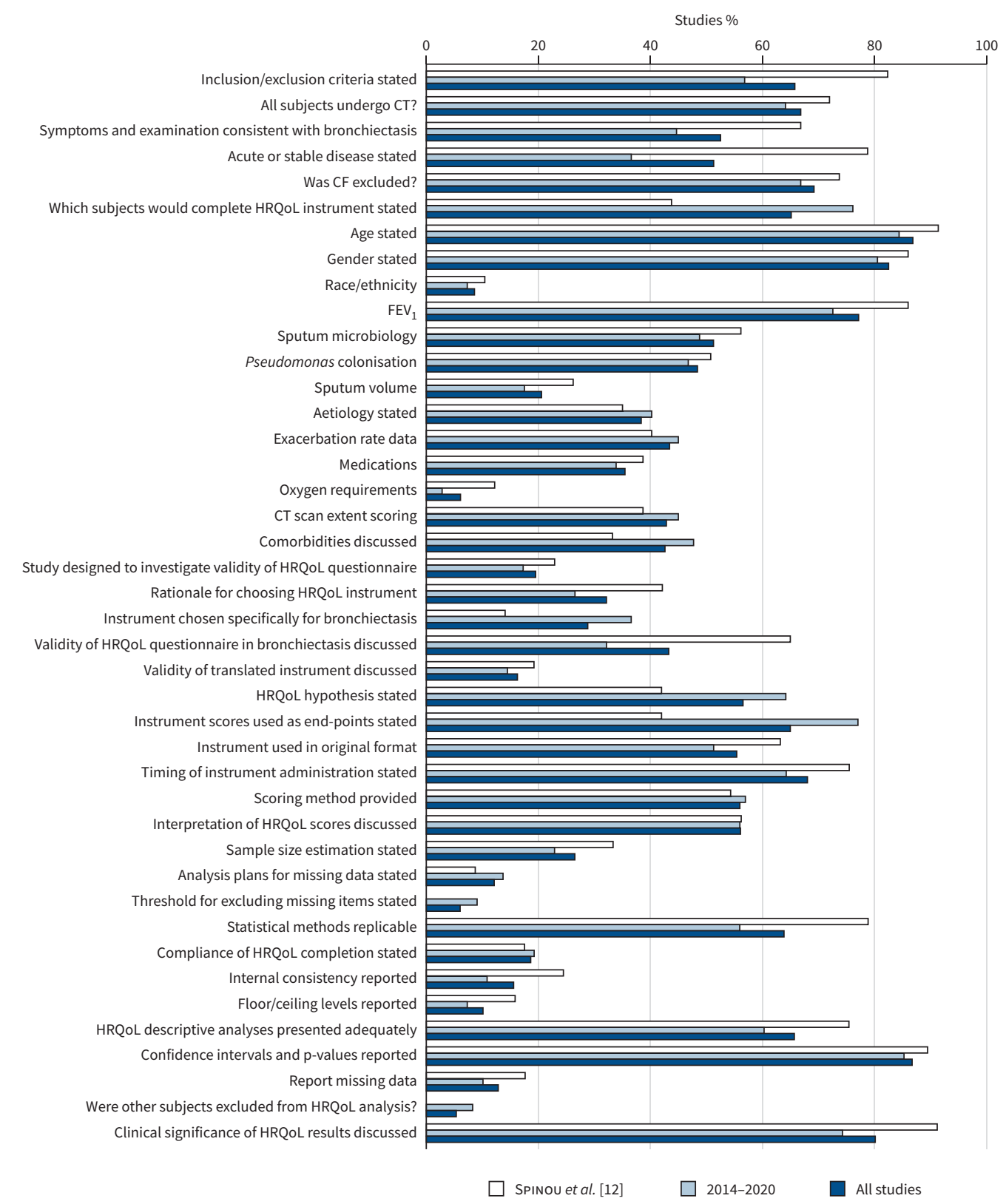

FIGURE 2 Quality of reporting of included studies. CT: computed tomography; CF: cystic fibrosis; FEV ${ }_{1}$ : forced expiratory volume in $1 \mathrm{~s}$; HRQoL: health-related quality of life; RCT: randomised controlled trial. $x$-axis: percentage of studies which meet each criterion; $y$-axis: quality criterion. Total studies, $n=166$ : SpINou et al. [12], $\mathrm{n}=57$ and 6 November 2014-31 December 2020, $\mathrm{n}=109$. SpINou et al. [12] reported on quality of studies included in meta-analysis of HRQoL with clinical associations and did not report on quality of other studies (i.e. RCTs assessing responsiveness). Number of studies reported by SpInou et al. [12], $n=37$ (additionally we have assessed the quality of $n=20$ RCTs reported on in the prior review which were included in the current meta-analysis).

figure E1. Overall, respiratory-specific questionnaires were the most widely used to assess HRQoL: SGRQ (58\%) and LCQ (32\%). The bronchiectasis-specific QOL-B was the third most commonly used (27\%). Mean HRQoL scores, where available, are presented in supplementary table E4. Translated questionnaires are outlined in appendix 5 in the supplementary material.

Internal consistency

The internal consistency for all questionnaires is detailed in table 1. BHQ, LCQ, CAT and SF-36 had good internal consistency in all domains reported (Cronbach's $\alpha \geqslant 0.7$ ) across all studies, and QOL-B, SGRQ, 
TABLE 1 Internal consistency and test-retest reliability of health-related quality of life (HRQoL) questionnaires

\begin{tabular}{|c|c|c|c|c|c|c|}
\hline \multirow[t]{2}{*}{ Reference $^{\#}$} & \multirow[t]{2}{*}{ HRQoL questionnaire } & \multicolumn{2}{|c|}{ Internal consistency } & \multicolumn{2}{|c|}{ Test-retest reliability } & \multirow[t]{2}{*}{ Timeframe } \\
\hline & & Domain & Cronbach's $\boldsymbol{\alpha}^{\S}$ & Domain & $\mathrm{ICC}^{\S}$ & \\
\hline \multicolumn{7}{|l|}{ Bronchiectasis-specific } \\
\hline Quittner (2010b) & QOL-B & 8 domains & $0.65-0.94$ & 8 domains & $0.72-0.88$ & 2 weeks \pm 3 days \\
\hline Quittner (2010a) & QOL-B & 8 domains & $0.73-0.96$ & 8 domains & NR & \\
\hline \multirow[t]{8}{*}{ Quittner (2014) } & QOL-B V3. $0^{+}$ & Respiratory symptoms & 0.82 & Respiratory symptoms & 0.80 & 2 weeks \\
\hline & & Physical functioning & 0.94 & Physical functioning & 0.88 & 2 weeks \\
\hline & & Vitality & 0.85 & Vitality & 0.67 & 2 weeks \\
\hline & & Role functioning & 0.86 & Role functioning & 0.84 & 2 weeks \\
\hline & & Health perceptions & 0.77 & Health perceptions & 0.78 & 2 weeks \\
\hline & & Emotional functioning & 0.72 & Emotional functioning & 0.82 & 2 weeks \\
\hline & & Social functioning & 0.66 & Social functioning & 0.85 & 2 weeks \\
\hline & & Treatment burden & 0.84 & Treatment burden & 0.76 & 2 weeks \\
\hline \multirow[t]{8}{*}{ Quittner (2015) } & QOL-B V3.0 & Respiratory symptoms & 0.81 & Respiratory symptoms & 0.83 & 2 weeks \\
\hline & & Physical functioning & 0.91 & Physical functioning & 0.85 & 2 weeks \\
\hline & & Vitality & 0.73 & Vitality & 0.74 & 2 weeks \\
\hline & & Role functioning & 0.84 & Role functioning & 0.86 & 2 weeks \\
\hline & & Health perceptions & 0.77 & Health perceptions & 0.76 & 2 weeks \\
\hline & & Emotional functioning & 0.83 & Emotional functioning & 0.79 & 2 weeks \\
\hline & & Social functioning & 0.77 & Social functioning & 0.80 & 2 weeks \\
\hline & & Treatment burden & 0.78 & Treatment burden & 0.76 & 2 weeks \\
\hline \multirow[t]{8}{*}{ Olveira (2014a) } & QOL-B V3.0 & Respiratory symptoms & 0.87 & Respiratory symptoms & 0.83 & 2 weeks \\
\hline & & Physical functioning & 0.91 & Physical functioning & 0.88 & 2 weeks \\
\hline & & Vitality & 0.82 & Vitality & 0.78 & 2 weeks \\
\hline & & Role functioning & 0.84 & Role functioning & 0.86 & 2 weeks \\
\hline & & Health perceptions & 0.71 & Health perceptions & 0.83 & 2 weeks \\
\hline & & Emotional functioning & 0.84 & Emotional functioning & 0.86 & 2 weeks \\
\hline & & Social functioning & 0.70 & Social functioning & 0.78 & 2 weeks \\
\hline & & Treatment burden & 0.72 & Treatment burden & 0.68 & 2 weeks \\
\hline Sokol (2019) & QOL-B (German) & 8 domains & $0.867-0.888$ & 8 domains & NR & \\
\hline \multirow[t]{8}{*}{ Speck (2018) } & QOL-B & Respiratory symptoms & 0.81 & Respiratory symptoms & 0.70 & \\
\hline & & Physical functioning & NR & Physical functioning & NR & \\
\hline & & Vitality & NR & Vitality & NR & \\
\hline & & Role functioning & NR & Role functioning & NR & \\
\hline & & Health perceptions & NR & Health perceptions & NR & \\
\hline & & Emotional functioning & NR & Emotional functioning & NR & \\
\hline & & Social functioning & NR & Social functioning & NR & \\
\hline & & Treatment burden & NR & Treatment burden & NR & \\
\hline Spinou (2018) & QOL-B & 8 domains & $0.46-0.90$ & 8 domains & NR & \\
\hline Liu (2019) & QOL-B & 8 domains & $>0.64$ & 8 domains & NR & \\
\hline \multirow[t]{8}{*}{ De Camargo (2020) } & QOL-B & Respiratory symptoms & 0.85 & Respiratory symptoms & 0.85 & $1-2$ weeks \\
\hline & & Physical functioning & 0.91 & Physical functioning & 0.91 & $1-2$ weeks \\
\hline & & Vitality & 0.58 & Vitality & 0.58 & $1-2$ weeks \\
\hline & & Role functioning & 0.70 & Role functioning & 0.70 & $1-2$ weeks \\
\hline & & Health perceptions & 0.77 & Health perceptions & 0.77 & $1-2$ weeks \\
\hline & & Emotional functioning & 0.91 & Emotional functioning & 0.91 & $1-2$ weeks \\
\hline & & Social functioning & 0.93 & Social functioning & 0.93 & $1-2$ weeks \\
\hline & & Treatment burden & 0.70 & Treatment burden & 0.70 & $1-2$ weeks \\
\hline Spinou (2017b) & $\mathrm{BHQ}$ & Total & 0.85 & Total & 0.89 & 2 weeks \\
\hline Spinou (2018) & $\mathrm{BHQ}$ & Total & 0.84 & Total & NR & \\
\hline Gissel (2020) & $\mathrm{BHQ}$ & Total & 0.739 & Total & NR & \\
\hline \multicolumn{7}{|l|}{ Respiratory-specific } \\
\hline \multirow[t]{4}{*}{ Wilson (1997a) } & SGRQ & Total & NR & Total & 0.97 & 2 weeks \\
\hline & & Symptoms & 0.90 & Symptoms & 0.93 & 2 weeks \\
\hline & & Activity & 0.89 & Activity & 0.98 & 2 weeks \\
\hline & & Impact & 0.92 & Impact & 0.94 & 2 weeks \\
\hline \multirow[t]{4}{*}{ Chan $(2002)^{\triangleleft}$} & SGRQ & Total & 0.92 & Total & 0.93 & 2 weeks \\
\hline & & Symptoms & 0.59 & Symptoms & 0.94 & 2 weeks \\
\hline & & Activity & 0.91 & Activity & 0.84 & 2 weeks \\
\hline & & Impact & 0.88 & Impact & 0.89 & 2 weeks \\
\hline
\end{tabular}




\begin{tabular}{|c|c|c|c|c|c|c|}
\hline \multirow[t]{2}{*}{ Reference $^{\#}$} & \multirow[t]{2}{*}{ HRQoL questionnaire } & \multicolumn{2}{|c|}{ Internal consistency } & \multicolumn{2}{|c|}{ Test-retest reliability } & \multirow[t]{2}{*}{ Timeframe } \\
\hline & & Domain & Cronbach's $\boldsymbol{\alpha}^{\S}$ & Domain & $\mathrm{ICC}^{\S}$ & \\
\hline \multirow[t]{4}{*}{ Martinez-Garcia (2005) } & SGRQ & Total & 0.90 & Total & NR & \\
\hline & & Symptoms & 0.81 & Symptoms & NR & \\
\hline & & Activity & 0.87 & Activity & NR & \\
\hline & & Impact & 0.81 & Impact & NR & \\
\hline \multirow[t]{4}{*}{ Speck (2018) } & SGRQ & Total & NR & Total & NR & \\
\hline & & Symptoms & 0.646 & Symptoms & NR & \\
\hline & & Activity & NR & Activity & NR & \\
\hline & & Impact & NR & Impact & NR & \\
\hline \multirow[t]{4}{*}{ Murray (2009b) $)^{\natural}$} & LCQ & Total & NR & Total & 0.96 & 6 months \\
\hline & & Physical & NR & Physical & NR & \\
\hline & & Psychological & NR & Psychological & NR & \\
\hline & & Social & NR & Social & NR & \\
\hline \multirow[t]{4}{*}{ Munoz $(2013)^{\natural}$} & LCQ & Total & 0.91 & Total & NR & \\
\hline & & Physical & 0.94 & Physical & NR & \\
\hline & & Psychological & 0.93 & Psychological & NR & \\
\hline & & Social & 0.93 & Social & NR & \\
\hline \multirow[t]{4}{*}{ Gao (2014b) } & LCQ (Mandarin) & Total & 0.93 & Total & 0.89 & 6 months \\
\hline & & Physical & 0.83 & Physical & 0.84 & 6 months \\
\hline & & Psychological & 0.88 & Psychological & 0.82 & 6 months \\
\hline & & Social & 0.82 & Social & 0.89 & 6 months \\
\hline \multirow[t]{4}{*}{ Munoz (2016) } & LCQ (Spanish) & Total & 0.91 & Total & 0.84 & 15 days \\
\hline & & Physical & 0.87 & Physical & 0.87 & 15 days \\
\hline & & Psychological & 0.87 & Psychological & 0.82 & 15 days \\
\hline & & Social & 0.86 & Social & 0.79 & 15 days \\
\hline Lee $(2012)^{\natural}$ & CAT & Total & 0.84 & Total & NR & \\
\hline Lanza (2018) & CAT & Total & 0.91 & Total & 0.84 & $7-10$ days \\
\hline $\begin{array}{l}\text { De la Rosa Carrillo } \\
\text { (2020) }\end{array}$ & CAT & Total & 0.86 & Total & 0.95 & 15 days \\
\hline Finch (2020) & CAT & Total & NR & Total & 0.88 & 4 weeks \\
\hline \multirow[t]{5}{*}{ Vodanovich (2015) } & CRDQ & Total & NR & Total & 0.82 & 9 weeks \\
\hline & & Dyspnoea & 0.76 & Dyspnoea & 0.85 & 9 weeks \\
\hline & & Fatigue & 0.85 & Fatigue & 0.69 & 9 weeks \\
\hline & & Emotional functioning & 0.94 & Emotional functioning & 0.83 & 9 weeks \\
\hline & & Mastery & 0.80 & Mastery & 0.77 & 9 weeks \\
\hline \multirow[t]{4}{*}{ Bulcun (2015) } & SOLQ (Turkish) & Physical functioning & 0.72 & Physical functioning & 0.83 & 2 weeks \\
\hline & & Emotional functioning & 0.91 & Emotional functioning & 0.71 & 2 weeks \\
\hline & & Coping skills & 0.74 & Coping skills & 0.81 & 2 weeks \\
\hline & & Treatment satisfaction & 0.62 & Treatment satisfaction & 0.65 & 2 weeks \\
\hline \multicolumn{7}{|l|}{ Generic } \\
\hline Guilemany (2006) & SF-36 & 8 domains & $0.75-0.91$ & 8 domains & NR & \\
\hline
\end{tabular}

ICC: intraclass correlation coefficient; QOL-B: Quality of Life-Bronchiectasis; BHQ: Bronchiectasis Health Questionnaire; SGRQ: St George's Respiratory Questionnaire; LCQ: Leicester Cough Questionnaire; CAT: Chronic Obstructive Pulmonary Disease (COPD) Assessment Test; CRDQ: Chronic Respiratory Disease Questionnaire; SOLQ: Seattle Obstructive Lung Disease Questionnaire; SF-36: Medical Outcomes Study 36-item Short-Form Health Survey; NR: not reported. \#: see the References list and supplementary material; ": studies reported in the prior review by SPINou et al. [12]; ${ }^{+}$: the repeatability of QOL-B V3.0 was not reported (table presents data from QOL-B V2.0); ${ }^{\S}$ : Cronbach's $\alpha$ and ICC $\geqslant 0.7$ are considered acceptable for HRQoL questionnaires.

CRDQ and SOLQ had good internal consistency in all domains in the majority of (but not all) studies. Internal consistency was not reported for SNOT-20, CQLQ, EQ-5D-5L and NHP.

Test-retest reliability

Test-retest reliability for all questionnaires is detailed in table 1. BHQ, SGRQ, LCQ and CAT had good test-retest reliability in all domains reported (ICC $\geqslant 0.7$ ) across all studies, and QOL-B, CRDQ and SOLQ had good test-retest reliability in all domains in the majority of (but not all) studies. The majority of studies reported test-retest reliability over 2 weeks; however, only a few studies reported test-retest reliability over a longer period of time (up to 6 months). Test-retest reliability was not reported for SNOT-20, CQLQ, SF-36, NHP and EQ-5D-5L. 


\section{Discriminant validity}

Discriminant validity for all questionnaires is detailed in supplementary table E5 and appendix 6 in the supplementary material. Disease-specific and respiratory-specific HRQoL questionnaires were most commonly able to discriminate patients based on demographics, disease severity, exacerbations and bacteriology, as well as a range of other symptoms. In terms of disease-specific questionnaires, QOL-B had the most data available and it was able to discriminate patients based on demographics, disease severity, exacerbation rate, sputum and bacteriology, signs and symptoms, adherence to treatment, and exercise capacity. BHQ had some data available and it was able to discriminate patients based on demographics, lung function, exacerbation rate and hospital admissions. In terms of respiratory-specific questionnaires, SGRQ had the most data available and it was able to discriminate based on demographics, disease severity, exacerbation rate, bacteriology, signs and symptoms, and exercise capacity. LCQ, CAT, CRDQ, SNOT-20 and SOLQ had some data available. Discriminant data were limited for generic HRQoL questionnaires. Discriminant validity was not reported for CQLQ and NHP.

\section{Associations between $H R Q O L$ and clinical measures}

The associations between HRQoL and clinical measures were evaluated in a meta-analysis. The results are summarised in table 2 and forest plots with subgroup analysis of each HRQoL questionnaire are presented in supplementary figures E2-E9 and indicate a high level of heterogeneity. The strongest associations, albeit moderate, were between HRQoL and cough $\left(\mathrm{r}=0.5, \mathrm{I}^{2}=66 \%\right)$, dyspnoea $\left(\mathrm{r}=0.5, \mathrm{I}^{2}=87 \%\right)$ and Pseudomonas aeruginosa colonisation $\left(\mathrm{r}=0.5, \mathrm{I}^{2}=99 \%\right)$. Moderate associations existed between HRQoL and wheeze $\left(\mathrm{r}=0.4, \mathrm{I}^{2}=0 \%\right)$, fatigue $\left(\mathrm{r}=0.4, \mathrm{I}^{2}=36 \%\right)$, exercise capacity $\left(\mathrm{r}=-0.4, \mathrm{I}^{2}=73 \%\right)$, anxiety $(\mathrm{r}=0.4$, $\mathrm{I}^{2}=59 \%$ ), depression ( $\left.\mathrm{r}=0.5, \mathrm{I}^{2}=72 \%\right)$ and sputum volume $\left(\mathrm{r}=0.4, \mathrm{I}^{2}=89 \%\right)$. Weak to moderate associations existed between HRQoL questionnaires and lung function $\left(\mathrm{r}=-0.3, \mathrm{I}^{2}=51-71 \%\right)$, bacteriology $(\mathrm{r}=0.2$, $\left.\mathrm{I}^{2}=44 \%\right)$, inflammatory markers $\left(\mathrm{r}=0.3, \mathrm{I}^{2}=13 \%\right)$, healthcare utilisation $\left(\mathrm{r}=0.3-0.4, \mathrm{I}^{2}=8-66 \%\right)$, disease severity ( $\left.\mathrm{r}=0.2-0.4, \mathrm{I}^{2}=0-97 \%\right)$, demographics $\left(\mathrm{r}=-0.2-0.2, \mathrm{I}^{2}=0-65 \%\right)$, oxygen saturation $(\mathrm{r}=-0.3$, $\left.\mathrm{I}^{2}=0 \%\right)$ and comorbidities $\left(\mathrm{r}=0.08, \mathrm{I}^{2}=0 \%\right)$.

\section{Associations between HRQoL questionnaires}

Multiple questionnaires were used in 34\% of studies and 21 studies directly assessed associations between the questionnaires (supplementary table E6). There was some exploration of correlations between the different questionnaires. The data available do not permit comprehensive comparisons; however, there were data suggesting strong correlations existed between bronchiectasis-specific and respiratory-specific questionnaires, but not between bronchiectasis-specific and generic questionnaires.

\section{Responsiveness}

The responsiveness analysis included 4153 patients. Interventions included mucoactives, long-term inhaled or oral antibiotics, inhaled corticosteroids, statin treatment, neutrophil elastase inhibitors, physiotherapy, exercise, nutritional supplements, self-management programmes and alternative medicines. The treatment and placebo effect estimates for SGRQ, LCQ, QOL-B, EQ-5D-5L and CAT are presented in figure 3a and b. The total combined effect estimates for SGRQ, LCQ, QOL-B, EQ-5D-5L, SF-36 and CAT are presented in figure 4 . The $\mathrm{I}^{2}$-values for the treatment, placebo and total combined effect sizes were equal to $89 \%$, $64 \%$ and $83 \%$, respectively, indicating a high level of heterogeneity (all $p<0.01$ ). There was evidence that the observed symmetry in the funnel plots was marginally significant at the $5 \%$ significance level for the treatment effect, statistically significant for the placebo effect and nonsignificant for the total combined effect (supplementary figures E10 and E11). The effect estimate for treatment was 0.36 (95\% CI 0.24 , 0.48). The treatment effect for the disease-specific QOL-B (all domains) was small (0.08 (95\% CI 0.02 , 0.13)). The treatment effects were highest for the respiratory-specific questionnaires SGRQ (0.64 (95\% CI $0.22,1.07))$, LCQ $(0.72$ (95\% CI $0.36,1.07))$ and CAT $(0.75$ (95\% CI $0.43,1.07))$. The treatment effect was significant for the generic questionnaire EQ-5D-5L $(0.42$ (95\% CI $0.03,0.80))$. The effect estimate for placebo was statistically significant, but the effect was weak $(0.09$ (95\% CI $0.01,0.16)$ ). The total combined effect size was 0.21 (95\% CI 0.14, 0.27). The treatment, placebo and total combined effect estimates presented cumulatively by year are shown in supplementary figure E12. The HRQoL questionnaires became less responsive over the years. Responsiveness has not been investigated for BHQ, CRDQ, CQLQ, SOLQ and NHP.

\section{Floor/ceiling effects and missing data}

Floor/ceiling effects were reported for QOL-B, BHQ, SGRQ, LCQ, CAT and CRDQ (appendix 7 in the supplementary material). All studies met the standards for floor effects ( $<15 \%$ participants) for all questionnaires. For QOL-B, four out of five studies failed to meet the standards for ceiling effects $(>15 \%$ participants). For LCQ, one out of two studies failed to meet the standards for ceiling effects ( $>15 \%)$. All 
TABLE 2 Correlations between health-related quality of life (HRQOL) questionnaires and clinical measures

\begin{tabular}{|c|c|c|c|c|c|}
\hline Clinical measures & $\begin{array}{c}\text { Studies } \\
\mathrm{n}\end{array}$ & $\begin{array}{l}\text { Overall } \\
\text { participants } \mathrm{n}\end{array}$ & Correlation $(95 \% \mathrm{Cl})$ & p-value & $\begin{array}{c}\mathrm{I}^{2} \text {-value } \\
\%\end{array}$ \\
\hline \multicolumn{6}{|l|}{ Symptoms } \\
\hline Cough & 7 & 577 & $0.524(0.404,0.626)$ & $<0.01$ & 66 \\
\hline Dyspnoea & 119 & 25953 & $0.491(0.425,0.551)$ & $<0.01$ & 87 \\
\hline Wheeze & 2 & 213 & $0.422(0.304,0.527)$ & $<0.01$ & 0 \\
\hline Fatigue & 4 & 182 & $0.424(0.231,0.585)$ & $<0.01$ & 36 \\
\hline Anxiety (including HADS) & 114 & 11289 & $0.430(0.352,0.502)$ & $<0.01$ & 59 \\
\hline Depression (including HADS) & 114 & 11295 & $0.455(0.362,0.538)$ & $<0.01$ & 72 \\
\hline \multicolumn{6}{|l|}{$\begin{array}{l}\text { Lung function/exercise } \\
\text { capacity }\end{array}$} \\
\hline FVC \% pred & 112 & 11656 & $-0.291(-0.360,-0.218)$ & $<0.01$ & 51 \\
\hline $\mathrm{FEV}_{1} \%$ pred & 235 & 7032 & $-0.309(-0.355,-0.260)$ & $<0.01$ & 52 \\
\hline Exercise capacity & 223 & 12079 & $-0.388(-0.464,-0.305)$ & $<0.01$ & 73 \\
\hline \multicolumn{6}{|l|}{ Sputum bacteriology } \\
\hline Bacteriology & 5 & 1387 & $0.207(0.129,0.281)$ & $<0.01$ & 44 \\
\hline $\begin{array}{l}\text { Pseudomonas aeruginosa } \\
\text { colonisation }\end{array}$ & 45 & 11713 & $0.497(0.017,0.791)$ & $<0.01$ & 99 \\
\hline Inflammatory markers & 112 & 11808 & $0.287(0.238,0.334)$ & $<0.01$ & 13 \\
\hline \multicolumn{6}{|l|}{ Healthcare utilisation } \\
\hline Infection/exacerbation rate & 120 & 36367 & $0.324(0.277,0.369)$ & $<0.01$ & 66 \\
\hline Hospital admissions rate & 45 & 12027 & $0.366(0.326,0.406)$ & $<0.01$ & 15 \\
\hline \multicolumn{6}{|l|}{ Disease severity } \\
\hline BSI & 120 & 26557 & $0.393(0.351,0.433)$ & $<0.01$ & 64 \\
\hline FACED & 510 & 11975 & $0.311(0.271,0.351)$ & $<0.01$ & 0 \\
\hline CT bronchiectasis score & 10 & 1880 & $0.341(0.052,0.578)$ & 0.02 & 97 \\
\hline $\mathrm{CT}$ lung zone & 2 & 142 & $0.233(0.069,0.385)$ & $<0.01$ & 0 \\
\hline \multicolumn{6}{|l|}{ Demographics } \\
\hline Age & 9 & 1497 & $0.167(0.109,0.225)$ & $<0.01$ & 16 \\
\hline Sex & 6 & 868 & $-0.200(-0.353,-0.037)$ & 0.02 & 65 \\
\hline BMI & 58 & 11413 & $-0.161(-0.211,-0.109)$ & $<0.01$ & 0 \\
\hline \multicolumn{6}{|l|}{ Other } \\
\hline Sputum volume & 69 & 14971 & $0.359(0.275,0.437)$ & $<0.01$ & 89 \\
\hline Oxygen saturation & 4 & 324 & $-0.345(-0.439,-0.244)$ & $<0.01$ & 0 \\
\hline Comorbidities & 2 & 815 & $0.085(0.016,0.153)$ & 0.02 & 0 \\
\hline
\end{tabular}

For the purpose of comparison, higher score indicates poorer HRQoL. $1^{2}=0 \%$ indicates no observed heterogeneity, while $\mathrm{I}^{2}>50 \%$ indicates substantial heterogeneity. HADS: Hospital Anxiety and Depression; FVC: forced vital capacity; FEV $_{1}$ : forced expiratory volume in $1 \mathrm{~s}$; BSI: Bronchiectasis Severity Index; FACED: FEV ${ }_{1}$, age, chronic colonisation, extension, dyspnoea; CT: computed tomography; BMI: body mass index.

other questionnaires met the standards for ceiling effects ( $<15 \%$ participants). Missing data were reported for QOL-B, BHQ, SGRQ and CAT.

Minimal clinically important difference

MCID for QOL-B was determined from a bronchiectasis population. MCID for CAT was determined from a bronchiectasis population and was similar to MCIDs reported in COPD. MCIDs for SGRQ, LCQ, CRDQ, CQLQ, SF-36 and EQ-5D-5L visual analogue scale (VAS) component were based on other disease populations and are commonly used in bronchiectasis clinical trials. MCIDs for BHQ, SOLQ, CQLQ, SNOT-20, EQ-5D-5L five-digit code/index value and NHP have not been investigated in bronchiectasis or other respiratory conditions (table 3).

\section{Discussion}

This systematic review with new synthesised data from 166 studies highlights the value in evaluating psychometric properties of HRQoL to inform the choice of questionnaires used in clinical trials.

The psychometric properties varied between questionnaires. Internal consistency and test-retest reliability were generally good for the majority of questionnaires. However, the timeframe explored for test-retest was found to be generally quite short ( $\leqslant 15$ days), except for LCQ (6 months) [30, 31], CAT (4 weeks) [32] and 


\begin{tabular}{|c|c|c|}
\hline \multicolumn{3}{|l|}{ a) Treatment effect } \\
\hline Study or subgroup & $\mathbf{N}$ & $\begin{array}{c}\text { Responsiveness } \\
(95 \% \mathrm{Cl})\end{array}$ \\
\hline \multicolumn{3}{|l|}{ SGRQ } \\
\hline Drobnic (2005) & 20 & $0.23(-0.41,0.87)$ \\
\hline Newall (2005) & 12 & $0.64(-0.22,1.50)$ \\
\hline Martinez-Garcia (2006) & 29 & $0.24(-0.29,0.76)$ \\
\hline Maa (2007) & 11 & $0.32(-0.57,1.20)$ \\
\hline Lavery (2011) & 32 & $0.46(-0.05,0.96)$ \\
\hline Liaw (2011) & 13 & $0.51(-0.30,1.32)$ \\
\hline Hernando (2012) & 37 & $0.07(-0.39,0.53)$ \\
\hline Mandal (2012) & 12 & $1.23(0.31,2.15)$ \\
\hline Nicolson (2012) & 20 & $0.58(-0.07,1.23)$ \\
\hline Bilton (2013) & 231 & $3.34(3.06,3.62)$ \\
\hline Diego (2013) & 16 & $2.55(1.57,3.53)$ \\
\hline Serisier (2013) (ORBIT-2) & 22 & $0.18(-0.42,0.79)$ \\
\hline Serisier (2013) (BLESS) & 59 & $0.45(0.08,0.82)$ \\
\hline Stockley (2013) & 22 & $0.43(-0.19,1.04)$ \\
\hline Bilton (2014) & 233 & $0.78(0.59,0.97)$ \\
\hline Haworth (2014) (wk 12) & 73 & $0.13(-0.20,0.46)$ \\
\hline Haworth (2014) (wk 26) & 73 & $0.43(0.10,0.76)$ \\
\hline Liu (2014) & 22 & $0.99(0.35,1.63)$ \\
\hline Munoz (2018) & 19 & $0.44(-0.19,1.08)$ \\
\hline Watz (2019) & 47 & $-0.01(-0.42,0.40)$ \\
\hline Atalay (2019) & 6 & $1.09(-0.25,2.44)$ \\
\hline Brockwell (2020) (12 mths) & 54 & $-0.11(-0.44,0.21)$ \\
\hline Hester (2020) (12 wks) & 30 & $0.19(-0.32,0.71)$ \\
\hline Total $(95 \% \mathrm{Cl})$ & & $0.64(0.22,1.07)$ \\
\hline \multicolumn{3}{|c|}{$\begin{array}{l}\text { Heterogeneity: } \tau^{2}=0.97 ; \chi^{2}=423.37, d f=22(p<0.01) ; 1^{2}=95 \% \\
\text { Test for overall effect: } Z=2.98(p<0.01)\end{array}$} \\
\hline \multicolumn{3}{|l|}{ LCQ } \\
\hline Mandal (2012) & 12 & $1.13(0.22,2.04)$ \\
\hline Nicolson (2012) & 20 & $2.99(2.05,3.93)$ \\
\hline Bilton (2013) & 231 & $0.28(0.09,0.46)$ \\
\hline Serisier (2013) (BLESS) & 59 & $0.39(0.02,0.76)$ \\
\hline Lee (2014) & 43 & $0.11(-0.31,0.54)$ \\
\hline Mandal (2014) & 30 & $0.35(-0.17,0.87)$ \\
\hline Abdelhalim (2016) & 15 & $1.71(0.84,2.58)$ \\
\hline Munoz (2018) & 19 & $0.51(-0.13,1.15)$ \\
\hline Ozalp (2019) & 23 & $0.35(-0.25,0.94)$ \\
\hline Zhong (2020) & 27 & $0.71(0.15,1.28)$ \\
\hline Total $(95 \%$ Cl) & & $0.72(0.36,1.07)$ \\
\hline \multicolumn{3}{|c|}{$\begin{array}{l}\text { Heterogeneity: } \tau^{2}=0.23 ; \chi^{2}=45.13, d f=9(p<0.01) ; 12=80 \% \\
\text { Test for overall effect: } Z=3.99(p<0.01)\end{array}$} \\
\hline QOL-B & & \\
\hline Quittner (2014) & 89 & $0.25(-0.05,0.55)$ \\
\hline Quittner (2015) (RS, AIR BX1) & 134 & $0.29(0.05,0.53)$ \\
\hline Quittner (2015) (PF, AIR BX1) & 134 & $-0.08(-0.32,0.16)$ \\
\hline Quittner (2015) (Vit, AIR BX1) & 134 & $0.02(-0.22,0.26)$ \\
\hline Quittner (2015) (RF, AIR BX1) & 134 & $-0.07(-0.32,0.17)$ \\
\hline Quittner (2015) (HP, AIR BX1) & 134 & $0.03(-0.21,0.27)$ \\
\hline Quittner (2015) (EF, AIR BX1) & 134 & $0.01(-0.23,0.25)$ \\
\hline Quittner (2015) (SF, AIR BX1) & 134 & $0.07(-0.17,0.31)$ \\
\hline Quittner (2015) (TB, AIR BX1) & 134 & $-0.12(-0.37,0.12)$ \\
\hline Quittner (2015) (RS, AIR BX2) & 136 & $0.47(0.22,0.71)$ \\
\hline Quittner (2015) (PF, AIR BX2) & 136 & $-0.06(-0.30,0.17)$ \\
\hline Quittner (2015) (Vit, AIR BX2) & 136 & $-0.03(-0.27,0.21)$ \\
\hline Quittner (2015) (RF, AIR BX2) & 136 & $-0.11(-0.35,0.13)$ \\
\hline Quittner (2015) (HP, AIR BX2) & 136 & $0.05(-0.19,0.29)$ \\
\hline Quittner (2015) (EF, AIR BX2) & 136 & $0.10(-0.14,0.34)$ \\
\hline Quittner (2015) (SF, AIR BX2) & 136 & $0.03(-0.21,0.26)$ \\
\hline Quittner (2015) (TB, AIR BX2) & 136 & $-0.24(-0.48,0.00)$ \\
\hline Dona (2018) (RF, 3 mths) & 14 & $0.02(-0.74,0.77)$ \\
\hline Dona (2018) (RF, 6 mths) & 14 & $0.08(-0.67,0.84)$ \\
\hline Dona (2018) (Vit, 3 mths) & 14 & $0.48(-0.28,1.25)$ \\
\hline Dona (2018) (Vit, 6 mths) & 14 & $0.44(-0.32,1.21)$ \\
\hline Dona (2018) (EF, 3 mths) & 14 & $0.40(-0.36,1.17)$ \\
\hline Dona (2018) (EF, 6 mths) & 14 & $0.27(-0.49,1.03)$ \\
\hline Dona (2018) (SF, 3 mths) & 14 & $0.18(-0.58,0.93)$ \\
\hline Dona (2018) (SF, 6 mths) & 14 & $0.13(-0.63,0.88)$ \\
\hline Dona (2018) (TB, 3 mths) & 14 & $0.22(-0.53,0.98)$ \\
\hline Dona (2018) (TB, 6 mths) & 14 & $0.31(-0.45,1.07)$ \\
\hline Dona (2018) (HP, 3 mths) & 14 & $0.06(-0.70,0.81)$ \\
\hline Dona (2018) (HP, 6 mths) & 14 & $0.20(-0.56,0.96)$ \\
\hline Dona (2018) (RS, 3 mths) & 14 & $0.44(-0.32,1.21)$ \\
\hline Dona (2018) (RS, 6 mths) & 14 & $0.36(-0.40,1.12)$ \\
\hline Olveira (2016) (PF, 3 mths) & 14 & $0.29(-0.47,1.05)$ \\
\hline Olveira (2016) (PF, 6 mths) & 14 & $0.34(-0.42,1.10)$ \\
\hline Hester (2020) (PF, 2 wks) & 30 & $0.13(-0.39,0.64)$ \\
\hline Hester (2020) (RF, 2 wks) & 30 & $0.29(-0.23,0.81)$ \\
\hline Hester (2020) (Vit, 2 wks) & 30 & $0.28(-0.24,0.80)$ \\
\hline Hester (2020) (EF, 2 wks) & 30 & $0.34(-0.18,0.86)$ \\
\hline Hester (2020) (SF, 2 wks) & 30 & $0.33(-0.19,0.85)$ \\
\hline Hester (2020) (TB, 2 wks) & 22 & $0.13(-0.47,0.74)$ \\
\hline Hester (2020) (HP, 2 wks) & 30 & $0.41(-0.11,0.93)$ \\
\hline Hester (2020) (RS, $2 \mathrm{wks})$ & 30 & $0.60(0.08,1.13)$ \\
\hline Hester (2020) (PF, 12 wks) & 30 & $-0.20(-0.72,0.32)$ \\
\hline Hester (2020) (RF, 12 wks) & 30 & $0.12(-0.39,0.64)$ \\
\hline Hester (2020) (Vit, 12 wks) & 30 & $-0.05(-0.56,0.47)$ \\
\hline
\end{tabular}

$\begin{array}{lll}\begin{array}{c}\text { Weight } \\ \%\end{array} & \begin{array}{l}\text { b) Placebo effect } \\ \text { Study or subgroup } \\ \text { (95\% CI) }\end{array} \\ \text { SGRQ } \\ \text { Drobnic (2005) } \\ \text { Martinez-Garcia (2006) } \\ \text { Maa (2007) } \\ 1.0\end{array}$

b) Placebo effect

Martinez-Garcia (2006)

Maa (2007)

Hernando (2012)

Mandal (2012)

Bilton (2013)

Diego (2013)

Serisier (2013) (BLESS)

Stockley (2013)

Haworth (2014) (wk 12)

Haworth $(20142012$

Brockwell (2020) (12 mths)

Total $(95 \% \mathrm{Cl})$

Heterogeneity: $\tau^{2}=0.14 ; \chi^{2}=78.67, d f=21$

Test for overall effect: $Z=1.95(p=0.05)$

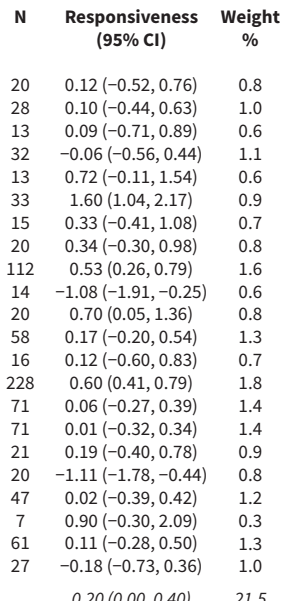

LCQ

Mandal (2012)
Nicolson (2012)
Bilton (2013)
Serisier (2013) (BLESS)
Lee (2014)
Mandal (2014)
Abdelhalim (2016)
Munoz (2018)
Ozalp (2019)
Zhong (2020)
Total (95\% Cl)

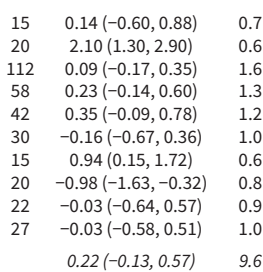

Heterogeneity: $\tau^{2}=0.23 ; \chi^{2}=41.29, d f=9(p<0.01) ; 12=78 \%$ Test for overall effect: $Z=1.24(p=0.22)$

\section{QOL-B}

Quittner (2015) (RS, AIR BX1)

Quittner (2015) (PF, AIR BX1)

Quittner (2015) (Vit, AIR BX1)

Quittner (2015) (RF, AIR BX1)

Quittner (2015) (HP, AIR BX1)

Quittner (2015) (EF, AIR BX1)

Quittner (2015) (SF, AIR BX1)

Quittner (2015) (TB, AIR BX1)

Quittner (2015) (RS, AIR BX2)

Quittner (2015) (PF, AIR BX2)

Quittner (2015)

Quittner (2015) (Nit, AR BX2)

Quittner (2015) (EF, AIR BX2)

Quittner (2015) (SF, AIR BX2)

Quittner (2015) (TB, AIR BX2)

Dona (2018) (RF, 3 mths)

Do (2018) (RF, 6 mths)

Dona (2018) (Rv, 6 mths)

Dona (2018) (Vit, 3 mths)

Dona (2018) (Vit, $6 \mathrm{mths})$

Dona (2018) (EF, 3 mths)

Dona (2018) (EF, 6 mths)

Dona (2018) (SF, 3 mths)

Dona (2018) (TB, 3 mths)

Dona (2018) (TB, 6 mths)

(HP, 3 mth

Dona

Dona (2018) (RS, 3 mths)

Dona (2018) (RS, 3 mths)

Dona (2018) (RS, 6 mths)

Olveira (2016) (PF, 3 mths)

Olveira (2016) (PF, 6 mths)

Hester (2020) (PF, 2 wks)

Hester (2020) (RF, 2 wks)

Hester (2020) (Vit, 2 wks)

Hester (2020) (EF, 2 wks)

Hester (2020) (SF, 2 wks)

Hester (2020) (TB, 2 wks)

Hester (2020) (HP, 2 wks)

Hester (2020) (RS, 2 wks)

Hester (2020) (PF, 12 wks)

Hester (2020) (RF, 12 wks)

Hester (2020) (Vit, 12 wks)

(

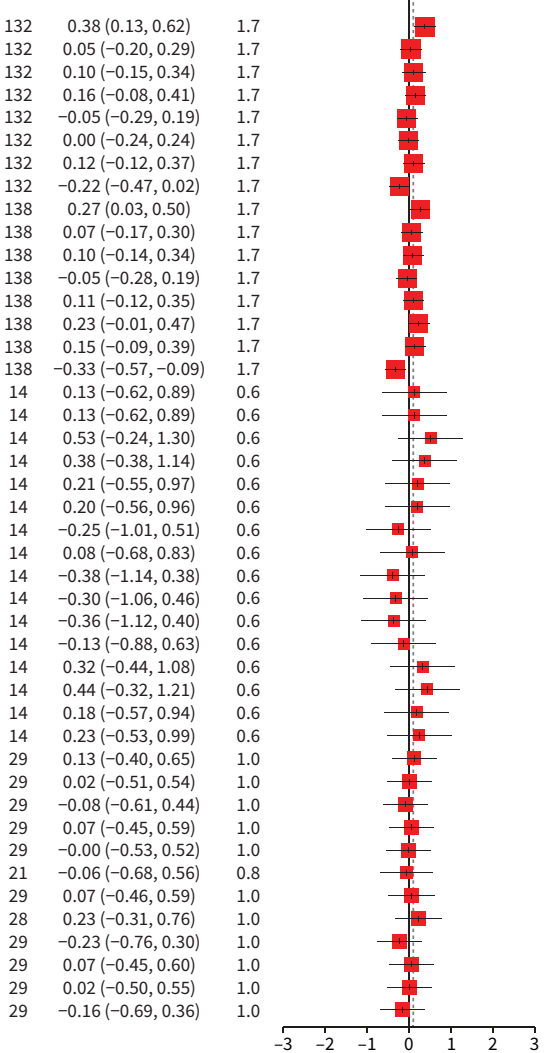

FIGURE 3 Continued on the following page. 


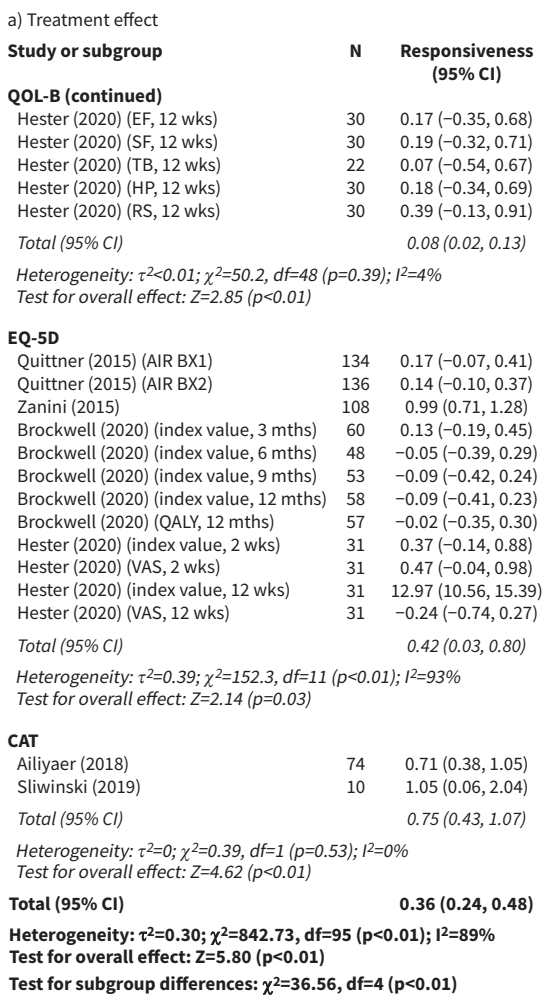

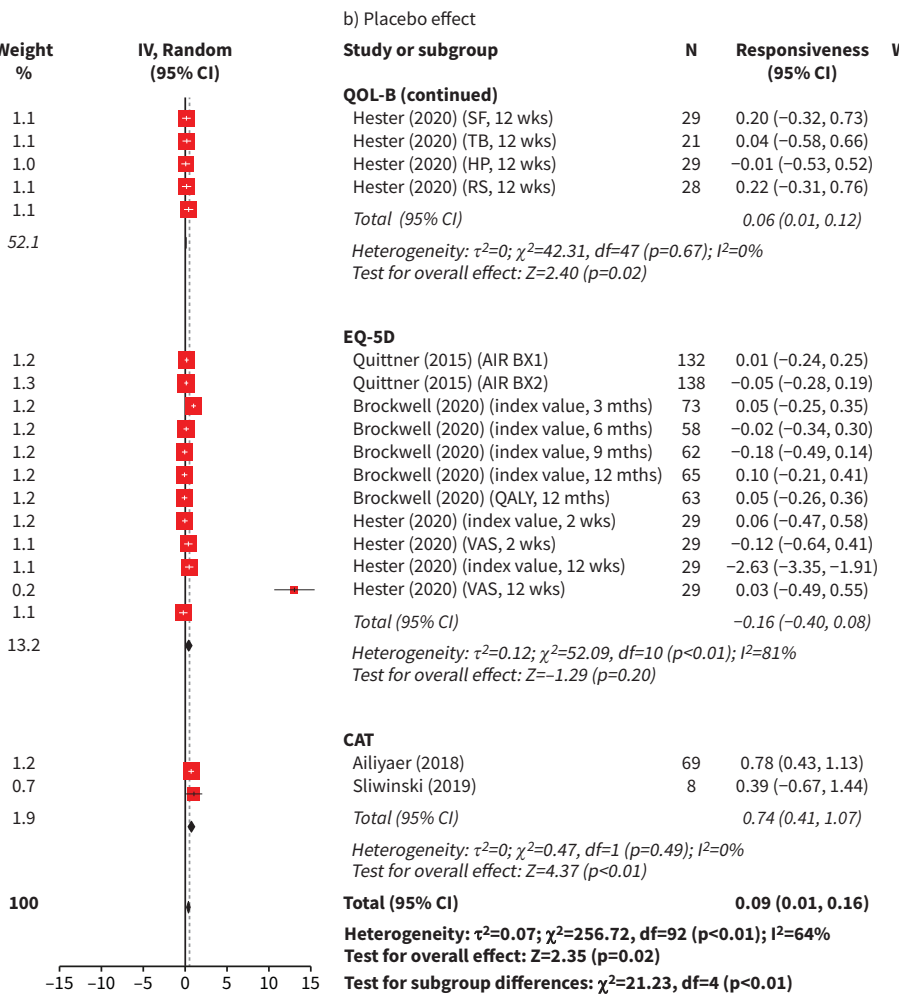

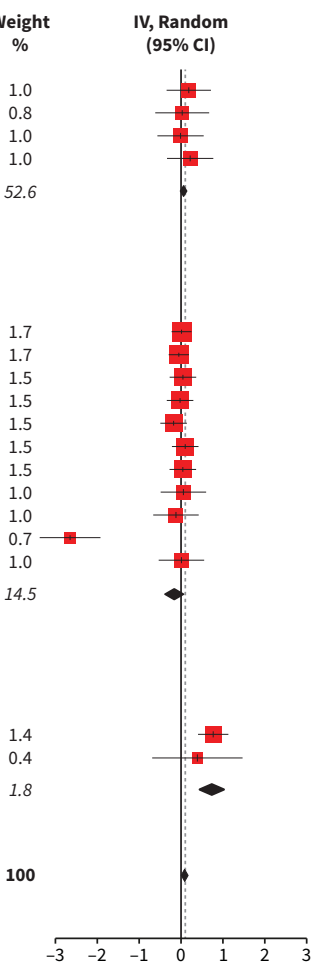

FIGURE 3 Forest plots for responsiveness of health-related quality of life (HRQoL) for a) treatment effect and b) placebo effect classified according to the HRQoL questionnaire: St George's Respiratory Questionnaire (SGRQ), Leicester Cough Questionnaire (LCQ), Quality of Life-Bronchiectasis (QOL-B), EuroQoL five-dimension (EQ-5D) and Chronic Obstructive Pulmonary Disease (COPD) Assessment Test (CAT). Total number of studies included in meta-analysis, $n=41$. The full list of studies is included in the References list and supplementary material. For HRQoL questionnaires where a lower score equates to better/improved HRQoL, scores were converted for meta-analysis. For all included questionnaires, positive effect sizes indicate improvement in HRQoL, while negative effect sizes indicate worsening of HRQoL. Higher score=better HRQoL. wk: week; mth: month; IV: interval variable; Random: random effect; RS: respiratory symptoms; PF: physical functioning; Vit: vitality; RF: role functioning; HP: health perceptions; EF: emotional functioning; SF: social functioning; TB: treatment burden; QALY: quality-adjusted life-years; VAS: visual analogue scale.

CRDQ (9 weeks) [33]. Given that the majority of randomised controlled trials are longer than 6 months, this is important to consider when selecting a HRQoL questionnaire. In ORBIT-3 and -4, QOL-B respiratory symptoms scores were not significantly different between baseline and 48 weeks in the treatment group [34]; however, they were significantly improved during on-treatment periods and these correlated with changes in bacterial load [3]. Future studies should investigate test-retest reliability of the HRQoL questionnaires over a longer timeframe in clinically stable patients and at multiple time-points which accurately reflect long-term variation in symptoms.

The HRQoL questionnaires were generally able to discriminate between demographics, important markers of clinical status and disease severity, as well as symptoms, highlighting that most questionnaires capture the impact of bronchiectasis on HRQoL.

The majority of HRQoL questionnaires were responsive, and the meta-analysis showed a difference between the treatment and placebo effect across the questionnaires. The effect sizes, categorised by HRQoL questionnaire, were higher in the respiratory-specific questionnaires (SGRQ, LCQ and CAT) compared with the bronchiectasis-specific QOL-B and this should be taken into consideration when selecting a HRQoL questionnaire for clinical trials. The lowest effect size was in the generic questionnaire SF-36.

Most recent studies used the QOL-B respiratory symptoms domain, which contains nine questions related to potentially important symptoms experienced by bronchiectasis patients as primary or secondary end-points. CRICHTON et al. [35] found that the QOL-B respiratory symptoms domain was unresponsive to inhaled antibiotic treatment, despite improvements in cough and sputum production. Other large randomised trials reported similar findings $[13,36]$. These studies highlight that responsiveness may 


Study or subgroup
SGRQ
Drobnic (2005)
Martinez-Garcia (2006)
Maa (2007)
Lavery (2011)
Liaw (2011)
Hernando (2012)
Mandal (2012)
Nicolson (2012)
Bilton (2013)
Diego (2013)
Serisier (2013) (ORBIT-2)
Serisier (2013) (BLESS)
Stockley (2013)
Bilton (2014)
Haworth (2014) (wk 12)
Haworth (2014) (wk 26)
Liu (2014)
Bedi (2017)
Aksamit (2018) (RS, CIPRO 14)
Aksamit (2018) (RS, CIPRO 28)
De Soyza (2018) (RS, CIPRO 14)
De Soyza (2018) (RS, CIPRO 28)
Munoz (2018)
Watz (2019)
Atalay (2019)
Brockwell (2020) (12 mths)
Hester (2020) (12 wks)
Total (95\% CI)

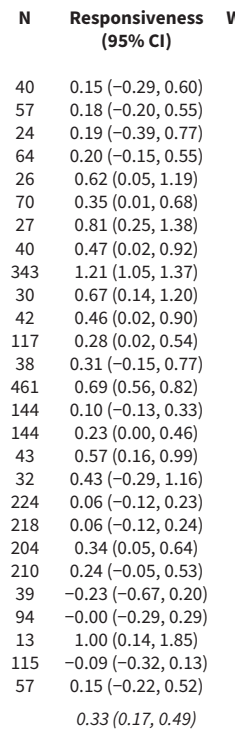

Heterogeneity: $\tau^{2}=0.14 ; \chi^{2}=198.06, d f=26(p<0.01) ; 1^{2}=87 \%$
Test for overall effect: $Z=4.04(p<0.01)$

LCQ

Mandal (2012)

Nicolson (2012)

Bilton (2013)

Serisier (2013) (BLESS)

Lee (2014)

Mandal (2014)

Abdelhalim (2016)

Bedi (2017)

Munoz (2018)

Ozalp (2019)

Zhong (2020)

Total (95\% Cl)

$27 \quad 0.68(0.12,1.24)$

$\begin{array}{cc}40 & 2.48(1.89,3.08) \\ 343 & 0.22(0.07,0.37)\end{array}$

$\begin{array}{ll}343 & 0.22(0.07,0.37) \\ 117 & 0.31(0.05,0.57)\end{array}$

$85 \quad 0.23(-0.07,0.54)$

$60 \quad 0.11(-0.25,0.47)$

$30 \quad 1.26(0.70,1.83)$

$32 \quad 0.27(-0.45,0.99)$

$39-0.06(-0.49,0.38)$

$45 \quad 0.13(-0.29,0.55)$

$54 \quad 0.53(0.14,0.92)$

$0.51(0.22,0.81)$

terogeneity: $\tau^{2}=0.19 ; \chi^{2}=71.21, d f=10(p<0.01) ; 1^{2}=86 \%$

Test for overall effect: $Z=3.47(p<0.01)$

QOL-B

Quittner (2015) (RS, AIR BX1)

Quittner (2015) (PF, AIR BX1)

Quittner (2015) (Vit, AIR BX1)

Quittner (2015) (RF, AIR BXI)

Quittner (2015) (HP, AIR BX1)

Quittner (2015) (EF, AIR BX1)

Quittner (2015) (EF, AIR BX1)

Uittner (2015) (TB, AIR BXI)

Quittner (2015) (RS, AIR BX2)

Quittner (2015) (PF, AIR BX2)

Quittner (2015) (Vit, AIR BX2)

Quittner (2015) (RF, AIR BX2)

Quittner (2015) (HP, AIR BX2)

Quittner (2015) (EF, AIR BX2)

Quittner (2015) (EF, AIR BX2)

Quittner (2015) (SF, AIR BX2)

Quittner (2015) (TB, AIR

Dona (2018) (RF, 3 mths)

Dona (2018) (RF, 6 mths)

Dona (2018) (Vit, 3 mths)

Dona (2018) (Vit, 6 mths)

Dona (2018) (EF, 3 mths)

Dona (2018) (EF, 6 mths)

Dona (2018) (SF, 3 mths)

Dona (2018) (SF, 6 mths)

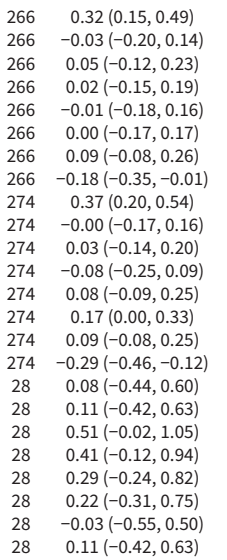

$\begin{gathered}\text { Weight } \\ \%\end{gathered}$
0.8
0.9
0.6
0.9
0.7
0.9
0.7
0.8
1.2
0.7
0.8
1.0
0.8
1.2
1.1
1.1
0.8
0.5
1.1
1.1
1.0
1.0
0.8
1.0
0.4
1.1
0.9
23.9

IV, Random

(95\% CI)

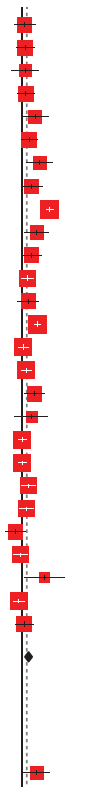

0.7
0.6
1.2
1.0
1.0
0.9
0.7
0.5
0.8
0.8
0.9
9.1

0.7
0.6
1.2
1.0
1.0
0.9
0.7
0.5
0.8
0.8
0.9
9.1

Study or subgroup

QOL-B (continued)

Dona (2018) (TB, 3 mths)

Dona (2018) (TB, 6 mths)

Dona (2018) (HP, 3 mths)

Dona (2018) (HP, 6 mths)

Dona (2018) (RS, 3 mths)

Dona (2018) (RS, $6 \mathrm{mths})$

Olveira (2016) (PF, 3 mths)

Olveira (2016) (PF, 3 mths

Olveira (2016) (PF, 6 mths)

Aksamit (2018) (RS, CIPRO 14)
Aksamit (2018) (RS, CIPRO 28)

Aksamit (2018) (RS, CIPRO 28)

De Soyza (2018) (RS, CIPRO 14)
De Soyza (2018) (RS, CIPRO 28)

De Soyza (2018) (RS, CIPRO
Hester (2020) (PF, 2 wks)

Hester (2020) (PF, 2 wks)
Hester (2020) (RF, 2 wks)

Hester (2020) (RF, 2 wks)
Hester (2020) (Vit, 2 wks)

Hester (2020) (Vit, 2 wks)
Hester (2020) (EF, 2 wks)

Hester (2020) (SF, 2 wks)

Hester (2020) (TB, 2 wks)

Hester (2020) (HP, 2 wks)

Hester (2020) (RS, 2 wks)

Hester (2020) (PF, 12 wks

Hester (2020) (RF, 12 wks)

Hester (2020) (RF, 12 wks)

Hester (2020) (Vit, 12 wks)

Hester (2020) (EF, 12 wks)

Hester (2020) (SF, 12 wks)
Hester (2020) (TB, 12 wks)

Hester (2020) (TB, 12 wks)
Hester (2020) (HP, 12 wks)

Hester (2020) (HP, 12 wks)
Hester (2020) (RS, 12 wks)

Total $(95 \%$ Cl)

Heterogeneity: $\tau^{2}<0.01 ; \chi^{2}=74.54, d f=51(p=0.02) ; 1^{2}=32 \%$

Test for overall effect: $Z=3.21(p<0.01)$

\section{EQ-5D}

Quittner (2015) (AIR BX1)

Quittner (2015) (AIR BX2)

Brockwell (2020) (index value, 3 mths) $\quad 133 \quad 0.11(-0.11,0.33)$

Brockwell (2020) (index value, 9 mths) $\quad 115-0.17(-0.40,0.06)$

Brockwell (2020) (index value, $12 \mathrm{mths}) 123 \quad 0.00(-0.22,0.22)$

Brockwell (2020) (QALY, 12 mths) $120 \quad 0.02(-0.21,0.24)$

$120 \quad 0.02(-0.21,0.24$

Hester (2020) (index value, 2 wks)

Hester (2020) (VAS, 2 wks)

Hester (2020) (index value, 12 wks)

Hester (2020) (VAS, 12 wks)

$60 \quad 0.17(-0.19,0.53)$

$60 \quad 0.17(-0.19,0.53)$

$\begin{array}{rr}60 & 4.84(4.12,5.56) \\ 60 & -0.10(-0.46,0.26)\end{array}$

Total $(95 \% \mathrm{Cl})$

$0.35(0.03,0.66)$

Heterogeneity: $\tau^{2}=0.25 ; \chi^{2}=176.6, d f=10(p<0.01) ; 12=94 \%$

Test for overall effect: $z=2.18(p=0.03)$

SF-36

Üzmezoğlu (2018) (GH)

Üzmezoğlu (2018) (PF)

Üzmezoğlu (2018) (PR)

Üzmezoğlu (2018) (ER)

Üzmezoğlu (2018) (SF)

Üzmezoğlu (2018) (Pain)

Üzmezoğlu (2018) (Vit)

Üzmezoğlu (2018) (MH)

Total $(95 \%$ Cl)

$36 \quad 0.07(-0.39,0.53)$

$\begin{array}{ll}36 & 0.22(-0.24,0.68)\end{array}$

$36 \quad 0.31(-0.15,0.77)$

$36 \quad 0.20(-0.25,0.66)$

$36-0.21(-0.67,0.24)$

$36 \quad 0.22(-0.24,0.68)$

$36-0.19(-0.65,0.26)$

$0.06(-0.10,0.22)$

政: $\tau^{2}=0 ; \chi^{2}=5.89, d f=7(p=0.55) ; 1^{2}=0 \%$

Test for overall effect: $Z=0.69(p=0.49)$

CAT

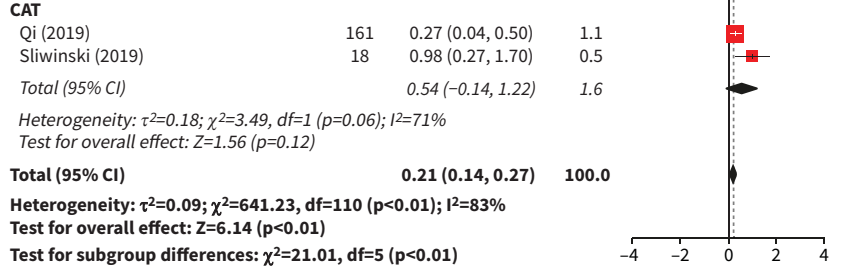

FIGURE 4 Forest plots for responsiveness of health-related quality of life (HRQoL) for total combined effect classified according to the HRQoL questionnaire: St George's Respiratory Questionnaire (SGRQ), Leicester Cough Questionnaire (LCQ), Quality of Life-Bronchiectasis (QOL-B), EuroQoL five-dimension (EQ-5D), Medical Outcomes Study 36-item Short-Form Health Survey (SF-36) and Chronic Obstructive Pulmonary Disease (COPD) Assessment Test (CAT). Total number of studies included in meta-analysis, $n=41$. The full list of studies is included in the References list and supplementary material. For HRQoL questionnaires where a lower score equates to better/improved HRQoL, scores were converted for meta-analysis. For all included questionnaires, positive effect sizes indicate improvement in HRQoL, while negative effect sizes indicate worsening of HRQoL. Higher score=better HRQoL. wk: week; mth: month; IV: interval variable; Random: random effect; RS: respiratory symptoms; PF: physical functioning; Vit: vitality; RF: role functioning; HP: health perceptions; EF: emotional functioning; SF: social functioning; TB: treatment burden; QALY: quality-adjusted life-years; VAS: visual analogue scale; GH: general health; PR: physical role; ER: emotional role; Pain: bodily pain; MH: mental health. 


\begin{tabular}{|c|c|c|c|}
\hline HRQoL questionnaire & $\begin{array}{l}\text { MCID study disease } \\
\text { population }\end{array}$ & MCID units & $\begin{array}{l}\text { Studies referenced as origin } \\
\text { of } \mathrm{MCID}^{\#}\end{array}$ \\
\hline \multirow[t]{4}{*}{ QOL-B } & Bronchiectasis & $\begin{array}{l}6.8 \text { (respiratory } \\
\text { symptoms) }\end{array}$ & Olveira (2014a) \\
\hline & Bronchiectasis & 7.0-10.0 & Quittner (2015) \\
\hline & Bronchiectasis & 8.6 & De Camargo (2020) \\
\hline & Bronchiectasis & $\begin{array}{l}\text { 8.1-8.3 (respiratory } \\
\text { symptoms) }\end{array}$ & Tong (2020) \\
\hline \multirow[t]{3}{*}{ SGRQ } & COPD & 4.0 & Jones (2005) \\
\hline & COPD & 5.8 & Schünemann (2003) \\
\hline & IPF & 7.0 & Swigris (2005) \\
\hline LCQ & Chronic cough & 1.3 & Raj (2009) \\
\hline \multirow[t]{2}{*}{ CQLQ } & IPF & $5.0-5.7$ & Lechtzin (2013) \\
\hline & Chronic cough & 10.6 & Fletcher (2010) \\
\hline \multirow[t]{4}{*}{ CAT } & COPD & $1.2-3.8$ & Kon (2013) \\
\hline & COPD & 3.5 & Zhou (2018) \\
\hline & Bronchiectasis & 3.0 & De la Rosa Carrillo (2020) \\
\hline & Bronchiectasis & $3.0-4.0$ & Finch $(2020)$ \\
\hline CRDQ & COPD & 0.5 & Jaeschke (1989) \\
\hline SF-36 & IPF & $2.0-4.0$ & Swigris (2005) \\
\hline EQ-5D VAS & COPD & 8.0 & Zanini (2015b) \\
\hline
\end{tabular}

QOL-B: Quality of Life-Bronchiectasis; SGRQ: St George's Respiratory Questionnaire; COPD: chronic obstructive pulmonary disease; IPF: idiopathic pulmonary fibrosis; LCQ: Leicester Cough Questionnaire; CQLQ: Cough Quality of Life Questionnaire; CAT: COPD Assessment Test; CRDQ: Chronic Respiratory Disease Questionnaire; SF-36: Medical Outcomes Study 36-item Short-Form Health Survey; EQ-5D: EuroQoL five-dimension; VAS: visual analogue scale. ": see the References list and supplementary material.

depend largely on the type of intervention and its ability to affect symptoms in specific domains of HRQoL questionnaires.

The reasons for an apparent decrease in the responsiveness of HRQoL questionnaires over time may be attributed to improved bronchiectasis management in recent years resulting in subtle, nonsignificant changes in outcome measures, such as HRQoL [37]. Establishment of the MCID is essential for clinical interpretation of HRQoL scores; however, only two questionnaires (QOL-B and CAT) have MCID values for bronchiectasis populations. Future studies need to address the MCID for the most commonly used HRQoL questionnaires, especially since large randomised trials for promising bronchiectasis treatments failed to demonstrate a significant change in HRQoL with QOL-B respiratory symptoms [13, 36], SGRQ [9, 13, 38, 39] and LCQ [39] despite evidence there were other clinically meaningful changes.

The findings for floor/ceiling effects are important for studies exploring interventions in early disease where HRQoL impairments may be less obvious and should be taken into consideration in such future studies.

Some studies used multiple questionnaires and this has resulted in challenges in interpretation, e.g. in RESPIRE-1 [13], and additional burden for patients. Multiple questionnaires may be valuable when they capture different aspects of HRQoL.

Our review has limitations. We did not contact study authors for unpublished data and we chose to limit the review to articles published in the English language only. Our methodology attempted to include bronchiectasis studies with homogenous populations. We recognise that $31 \%$ of studies included in our review did not require a radiological confirmation of bronchiectasis; however, they required a clinician diagnosis and symptoms consistent with bronchiectasis. The presence of heterogeneity in our meta-analysis results was therefore expected given the differences in the methodology, study population, sample size and study quality. Our methodology assumes that the interventions used in clinical trials are effective compared with placebo and also that the specific domain captured the effect of the intervention. Ideally, a positive control is needed to assess responsiveness, e.g. an acute exacerbation of bronchiectasis known to affect all domains of HRQoL questionnaires. This has been explored in a small number of studies only [30, 40-42] 
and this will be an important focus in future clinical trials. A limitation of HRQoL questionnaires is that they generate aggregate scores to represent distinct symptoms, e.g. cough, dyspnoea and chest pain, and not all may improve with a therapy. A recent study by CRICHTon et al. [35] illustrated this by showing that with QOL-B, inhaled antibiotics showed marked improvements in cough, sputum production and sputum colour, but there were no changes in any other symptoms.

This review considers the psychometric properties; however, the patient perspective is also key in the selection of HRQoL questionnaires. In cystic fibrosis, recent studies have involved patients and their caregivers in the assessment and improvement of PROs [43]. In bronchiectasis, DudGeON et al. [44] explored the patient perspective of four HRQoL questionnaires (QOL-B, SGRQ, LCQ and CAT). The authors concluded that bronchiectasis symptoms are highly individual and HRQoL tools do not fully capture the burden of disease. This is beginning to be explored by CRICHTON et al. [45] through the development of a novel PRO measure, i.e. the Bronchiectasis Impact Measure (BIM). Future studies of HRQoL tools in bronchiectasis should involve patients and their caregivers.

\section{Recommendations for future research}

Future studies should focus specifically on the medium- to long-term test-retest reliability, responsiveness and MCID in HRQoL questionnaires which show the best potential in bronchiectasis, such as QOL-B, BHQ and SGRQ. Future studies should focus on the involvement of patients and their caregivers in the assessment and improvement of these HRQoL questionnaires.

\section{Conclusions}

The consideration of psychometrics properties of HRQoL questionnaires is an important component of decision making to ensure optimal choice of HRQoL questionnaires in clinical trials. SGRQ was the most widely used HRQoL questionnaire in bronchiectasis studies and it had good psychometric properties; however, good psychometric data are emerging on bronchiectasis-specific HRQoL questionnaires such as QOL-B and BHQ.

Acknowledgement: We acknowledge the BronchUK consortium (www.bronch.ac.uk) supported by a Medical Research Council grant (MR/L011263/1).

This study is registered at PROSPERO with identifier number CRD42019146181.

Conflict of interest: R.H. McLeese has nothing to disclose. A. Spinou is the developer of the Bronchiectasis Health Questionnaire, one of the questionnaires included in this review. Z. Alfahl has nothing to disclose. M. Tsagris has nothing to disclose. J.S. Elborn reports grants from IMI/European Commission Inhaled Antibiotics in Bronchiectasis and Cystic Fibrosis (iABC), Novartis, Polyphor and Alexia, outside the submitted work. J.D. Chalmers reports grants and personal fees from AstraZeneca, Boehringer Ingelheim, Gilead Sciences, GlaxoSmithKline and Novartis, personal fees from Chiesi, Zambon and Janssen, outside the submitted work. A. De Soyza reports other (meeting support) from AstraZeneca, nonfinancial support (in-kind research support) from Novartis, nonfinancial support (meeting support) from Forest labs, personal fees for lectures and advisory board work from Bayer, personal fees for advisory board work from Novartis, other (travel bursary) from Chiesi, Almirall and Boehringer Ingelheim, grants and personal fees for lectures and advisory board work from AstraZeneca, grants from Pfizer, outside the submitted work; has received medical education grant support for a UK bronchiectasis network from GlaxoSmithKline, Gilead, Chiesi and Forest labs; A. De Soyza's employing institution receives fees for his work as coordinating investigator in a phase III trial in bronchiectasis sponsored by Bayer. M.R. Loebinger reports personal fees from Insmed, AstraZeneca and Grifols, outside the submitted work. S.S. Birring reports grants and personal fees from Merck, personal fees from Bayer, Shionogi and Bellus, outside the submitted work; and has a patent BHQ pending, and a patent LCQ with royalties paid. K.C. Fragkos has nothing to disclose. R. Wilson has nothing to disclose. K. O’Neill has nothing to disclose. J.M. Bradley has nothing to disclose.

\section{References}

1 Hill AT, Sullivan AL, Chalmers JD, et al. British Thoracic Society guideline for bronchiectasis in adults. Thorax 2019; 74: Suppl. 1, 1-69.

2 Polverino E, Goeminne PC, McDonnell MJ, et al. European Respiratory Society guidelines for the management of adult bronchiectasis. Eur Respir J 2017; 50: 1700629.

3 Chalmers JD, Cipolla D, Thompson B, et al. Changes in respiratory symptoms during 48 weeks treatment with ARD-3150 (inhaled liposomal ciprofloxacin) in bronchiectasis: results from the ORBIT-3 and -4 studies. Eur Respir J 2020; 56: 2000110. 
4 Chalmers JD, Haworth CS, Metersky ML, et al. Phase 2 trial of the DPP-1 inhibitor brensocatib in bronchiectasis. N Engl J Med 2020; 383: 2127-2137.

5 Loebinger MR, Polverino E, Chalmers JD, et al. Efficacy and safety of TOBI Podhaler in Pseudomonas aeruginosa infected bronchiectasis patients: iBEST study. Eur Respir J 2021; 57: 2001451.

6 Wong C, Jayaram L, Karalus N, et al. Azithromycin for prevention of exacerbations in non-cystic fibrosis bronchiectasis (EMBRACE): a randomised, double-blind, placebo-controlled trial. Lancet 2012; 380: 660-667.

7 Bilton D, Tino G, Barker AF, et al. Inhaled mannitol for non-cystic fibrosis bronchiectasis: a randomised, controlled trial. Thorax 2014; 69: 1073.

8 Haworth CS, Foweraker JE, Wilkinson P, et al. Inhaled colistin in patients with bronchiectasis and chronic Pseudomonas aeruginosa infection. Am J Respir Crit Care Med 2014; 189: 975-982.

9 Wilson R, Welte T, Polverino E, et al. Ciprofloxacin dry powder for inhalation in non-cystic fibrosis bronchiectasis: a phase II randomised study. Eur Respir J 2013; 41: 1107-1115.

10 Food and Drug Administration. Patient-reported Outcome Measures: Use in Medical Product Development to Support Labeling Claims. Silver Spring, FDA, 2009.

11 European Medicines Agency. Regulatory science strategy to 2025: human stakeholder workshop. 2019. www. ema.europa.eu/en/events/multi-stakeholder-workshop-draft-regulatory-science-2025-strategy-stakeholdershuman-medicines Date last accessed: 14 June 2021.

12 Spinou A, Fragkos KC, Lee KK, et al. The validity of health-related quality of life questionnaires in bronchiectasis: a systematic review and meta-analysis. Thorax 2016; 71: 683-694.

13 De Soyza A, Aksamit T, Bandel TJ, et al. RESPIRE 1: a phase III placebo-controlled randomised trial of ciprofloxacin dry powder for inhalation in non-cystic fibrosis bronchiectasis. Eur Respir J 2018; 51: 1702052.

14 Moher D, Liberati A, Tetzlaff $\mathrm{J}$, et al. Preferred reporting items for systematic reviews and meta-analyses: the PRISMA statement. PLoS Med 2009; 6: e1000097.

15 Scottish Intercollegiate Guidelines Network. SIGN Methodology checklist 1: systematic reviews and meta-analyses. 2020. www.sign.ac.uk/media/1721/srchecklist.doc Date last accessed: 14 June 2021.

16 Bradley JM, Madge S, Morton AM, et al. Cystic fibrosis research in allied health and nursing professions. J Cyst Fibros 2012; 11: 387-392.

17 Kent L, Reix P, Innes JA, et al. Lung clearance index: evidence for use in clinical trials in cystic fibrosis. J Cyst Fibros 2014; 13: 123-138.

18 Swigris JJ, Kuschner WG, Jacobs SS, et al. Health-related quality of life in patients with idiopathic pulmonary fibrosis: a systematic review. Thorax 2005; 60: 588-594.

19 Portney L. Concepts of measurement reliability. In: Foundations of Clinical Research: Applications to Evidence-based Practice. 4th Edn. Philadelphia, F.A. Davis, 2020; pp. 115-127.

20 Aaronson N, Alonso J, Burnam A, et al. Assessing health status and quality-of-life instruments: attributes and review criteria. Qual Life Res 2002; 11: 193-205.

21 Rosenthal R, Rosnow RL, Rubin DB. Contrasts and Effect Sizes in Behavioral Research: A Correlational Approach. Cambridge, Cambridge University Press, 1999.

22 Ailiyaer $\mathrm{Y}$, Wang $\mathrm{X}$, Zhang $\mathrm{Y}$, et al. A prospective trial of nebulised amikacin in the treatment of bronchiectasis exacerbation. Respiration 2018; 95: 327-333.

23 Wang $\mathrm{H}$, Ji XB, Mao B, et al. Pseudomonas aeruginosa isolation in patients with non-cystic fibrosis bronchiectasis: a retrospective study. BMJ Open 2018; 8: e014613.

24 Courtney JM, Kelly M, Watt A, et al. Quality of life and inflammation in exacerbations of bronchiectasis. Chron Respir Dis 2008; 5: 161-168.

25 Altenburg J, Wortel K, de Graaff CS, et al. Validation of a visual analogue score (LRTI-VAS) in non-CF bronchiectasis. Clin Respir J 2016; 10: 168-175.

26 Murray MP, Turnbull K, Macquarrie S, et al. Assessing response to treatment of exacerbations of bronchiectasis in adults. Eur Respir J 2009; 33: 312-318.

27 Abdelhalim HA, Aboeinaga HH, Fathy KA. Comparison between active cycles of breathing with postural drainage versus conventional chest physiotherapy in subjects with bronchiectasis. Egypt J Chest Dis Tuberc 2016; 65: 157-165.

28 Sliwinski P, Klatka D, Gladzka A, et al. Benefits of Simeox airway clearance technology in non-cystic fibrosis patients with bronchiectasis. Am J Respir Crit Care Med 2019; 201: A5720.

29 Varughese PG. Assessment of bronchiectasis severity using FACED score and Bronchiectasis Severity Index and its correlation with quality of life indices. Chest 2020; 158: 4 Suppl., A2448.

30 Gao YHY, Guan WJ, Xu G, et al. Validation of the Mandarin Chinese version of the Leicester Cough Questionnaire in bronchiectasis. Int J Tuberc Lung Dis 2014; 18: 1431-1437.

31 Murray MP, Turnbull K, MacQuarrie S, et al. Validation of the Leicester Cough Questionnaire in non-cystic fibrosis bronchiectasis. Eur Respir J 2009; 34: 125-131.

32 Finch S, Laska IF, Abo-Leyah $\mathrm{H}$, et al. Validation of the COPD assessment test (CAT) as an outcome measure in bronchiectasis. Chest 2020; 157: 815-823. 
33 Vodanovich DA, Bicknell TJ, Holland AE, et al. Validity and reliability of the chronic respiratory disease questionnaire in elderly individuals with mild to moderate non-cystic fibrosis bronchiectasis. Respiration 2015; 90: 89-96.

34 Haworth CS, Bilton D, Chalmers JD, et al. Inhaled liposomal ciprofloxacin in patients with non-cystic fibrosis bronchiectasis and chronic lung infection with Pseudomonas aeruginosa (ORBIT-3 and ORBIT-4): two phase 3 , randomised controlled trials. Lancet Respir Med 2019; 7: 213-226.

35 Crichton ML, Lonergan M, Barker AF, et al. Inhaled aztreonam improves symptoms of cough and sputum production in patients with bronchiectasis: a post-hoc analysis of the AIR-BX studies. Eur Respir J 2020; 56 : 2000608.

36 Aksamit T, De Soyza A, Bandel TJ, et al. RESPIRE 2: a phase III placebo-controlled randomised trial of ciprofloxacin dry powder for inhalation in non-cystic fibrosis bronchiectasis. Eur Respir J 2018; 51: 1702053.

37 Clarke M, Brice A, Chalmers I. Accumulating research: a systematic account of how cumulative meta-analyses would have provided knowledge, improved health, reduced harm and saved resources. PLoS One 2014; 9: e102670.

38 Serisier DJ, Bilton D, De Soyza A, et al. Inhaled, dual release liposomal ciprofloxacin in non-cystic fibrosis bronchiectasis (ORBIT-2): a randomised, double-blind, placebo-controlled trial. Thorax 2013; 68: 812-817.

39 Serisier DJ, Martin MI, McGuckin MA, et al. Effect of long-term, low-dose erythromycin on pulmonary exacerbations among patients with non-cystic fibrosis bronchiectasis: the BLESS randomised controlled trial. JAMA 2013; 309: 1260-1267.

40 Chalmers JD, Crichton ML, Brady G, et al. Pulmonary rehabilitation after exacerbation of bronchiectasis: a pilot randomised controlled trial. BMC Pulm Med 2019; 19: 85.

41 De la Rosa Carrillo D, Olveira C, Garcia-Clemente M, et al. COPD Assessment Test in bronchiectasis: minimum clinically important difference and psychometric validation: a prospective study. Chest 2020; 157: 824-833.

42 Guan WJ, Gao YH, Xu G, et al. Inflammatory responses, spirometry, and quality of life in subjects with bronchiectasis exacerbations. Respir Care 2015; 60: 1180-1189.

43 Hayes K, Sermet I, Bontemps V, et al. "Il faut continuer à poser des questions/We must continue to ask questions." Patient-reported outcomes in cystic fibrosis: a qualitative study of patients with cystic fibrosis and their caregivers. J Cyst Fibros 2020; 19: Suppl. 2, S51.

44 Dudgeon EK, Crichton M, Chalmers JD. "The missing ingredient": the patient perspective of health related quality of life in bronchiectasis: a qualitative study. BMC Pulm Med 2018; 18: 81.

45 Crichton ML, Dudgeon EK, Shoemark A, et al. Validation of the bronchiectasis impact measure (BIM): a novel patient-reported outcome measure. Eur Respir J 2021; 57: 2003156. 